\title{
COMMENTS
}

\section{Comments on the special issue on Agri-product Quality and Safety}

\author{
Charles F. SHOEMAKER (凶) \\ Department of Food Science and Technology, University of California, Davis, CA 95616, USA
}

Since the beginning of the industrial revolution about two hundred years ago there has been rapid development of globalization in the world. Today, globalization continues at an increasing pace, and more countries and their peoples are able to benefit from this development. A key foundation of global development is advances in the world's agricultural and food systems.

Increasingly, the world's consumer demand safe, nutritious and quality foods. The challenges to meeting these demands during rapid global development are explored from various perspectives in this issue. Dr. Jen presents a case study of China and the diversity of problems in the world's largest country. The remaining papers examine various aspects of emerging problems in the world's food systems. Different food safety issues are discussed ranging from microbial, chemical and biochemical issues, several nutritional fields are also addressed such as the effectiveness of the digestive process.

The diversity of topics presented in this special issue represents the breadth of challenges facing the international food science community. All must be tackled with integrative approaches to provide a solid foundation for the rapid global developments which are bringing many positive benefits to the world's standard of living.

Received June 1, 2018; accepted June 15, 2018

Correspondence: cfshoemaker@ucdavis.edu

(C) The Author(s) 2018. Published by Higher Education Press. This is an open access article under the CC BY license (http://creativecommons.org/licenses/by/4.0) 